\title{
Electrophysiological evidence for abnormal glutamate-GABA association following psychosis onset
}

\author{
Daisuke Koshiyama', Kenji Kirihara', Mariko Tada',2, Tatsuya Nagai ${ }^{1,3}$, Mao Fujioka', Eriko Ichikawa', Kazusa Ohta', \\ Motoko Tani ${ }^{1}$, Maiko Tsuchiya ${ }^{1}$, Akiko Kanehara', Kentaro Morita', Kingo Sawada (1)', Jun Matsuoka', \\ Yoshihiro Satomura', Shinsuke Koike $\mathbb{1}^{1,2,4,5}$, Motomu Suga ${ }^{1,6}$, Tsuyoshi Araki ${ }^{1}$ and Kiyoto Kasai ${ }^{1,2}$
}

\begin{abstract}
Previous studies have shown glutamatergic dysfunction and $\gamma$-aminobutyric acid (GABA)-ergic dysfunction in schizophrenia. Animal studies suggest that N-methyl-D-aspartate receptor (NMDAR) dysfunction and GABA-ergic dysfunction interact with each other and lead to alterations in excitatory/inhibitory balance. The NMDAR and GABAergic-interneuron functions may be indexed by mismatch negativity (MMN) and auditory steady-state gammaband response (ASSR), respectively. However, no previous studies have tested the hypothesis of an abnormal association between MMN and gamma-band ASSR in the same patients to identify the in vivo evidence of NMDARGABA association during the early stages of psychosis. Participants were individuals with recent-onset schizophrenia (ROSZ; $N=21)$, ultra-high risk (UHR; $N=27)$, and healthy controls $(H C s ; N=24)$. The MMN amplitude was significantly impaired in ROSZ ( $p=0.001, d=1.20)$ and UHR $(p=0.003, d=1.01)$ compared with HCs. The intertrial phase coherence (ITC) index of gamma-band ASSR was significantly reduced in ROSZ compared with HCs $(p<0.001, d=-$ 1.27) and UHR ( $p=0.032, d=-0.75)$. The event-related spectral perturbation (ERSP) index of gamma-band ASSR was significantly smaller in ROSZ compared with $\mathrm{HCs}(p<0.001, d=-1.21)$. The MMN amplitude was significantly correlated with the ITC in ROSZ $(r=-0.69, p<0.001)$. These findings provide the first in vivo evidence that an abnormal association of the electrophysiological indices of NMDAR and GABA dysfunctions may be present in recentonset schizophrenia.
\end{abstract}

\section{Introduction}

Glutamatergic dysfunction is considered to be one of the major hypotheses for the pathophysiology of schizophrenia $^{1-3}$. In an initial clinical study, Krystal et al. ${ }^{4}$ showed that ketamine, an antagonist of N-methyl-Daspartate receptors (NMDAR: a glutamate receptor), induces schizophrenia-like symptoms in healthy volunteers. Subsequent studies have shown abnormal

\footnotetext{
Correspondence: Kiyoto Kasai (kasaik-tky@umin.net)

${ }^{1}$ Department of Neuropsychiatry, Graduate School of Medicine, The University

of Tokyo, 7-3-1, Hongo, Bunkyo-ku, Tokyo 113-8655, Japan

${ }^{2}$ The International Research Center for Neurointelligence (WPI-IRCN) at The University of Tokyo Institutes for Advanced Study (UTIAS), The University of Tokyo, 7-3-1, Hongo, Bunkyo-ku, Tokyo 113-8655, Japan

Full list of author information is available at the end of the article.
}

metabolites of glutamatergic amino acids, which are coagonists of NMDAR, in the plasma or cerebrospinal fluid $(\mathrm{CSF})^{5,6}$, and in the anterior cingulate cortex (ACC) using proton magnetic resonance spectroscopy $\left({ }^{1} \mathrm{H}-\mathrm{MRS}\right)^{7,8}$ in patients with schizophrenia. In contrast, investigations on postmortem brains of patients with schizophrenia have revealed abnormalities in $\gamma$-aminobutyric acid (GABA)ergic interneurons, such as a reduced expression of GABA-synthesizing enzyme glutamic acid decarboxylase 67 (GAD67) and parvalbumin (PV) in the cortical neurons $^{9-11}$. Furthermore, clinical studies have shown a reduction of GABA in the ACC measured by ${ }^{1} \mathrm{H}$-MRS in chronic schizophrenia ${ }^{12}$ and in first-episode schizophrenia ${ }^{13}$. 
Table 1 Demographics of participants

\begin{tabular}{|c|c|c|c|c|}
\hline & ROSZ & UHR & $\mathrm{HC}$ & Statistics \\
\hline$N(\text { sex ratio } M / F)^{a}$ & $21(11 / 10)$ & $27(14 / 13)$ & $24(10 / 14)$ & $x^{2}=0.696, d f=2, p=0.706$ \\
\hline Age $\left(\right.$ years) ${ }^{b}$ & $24.0(6.7)$ & $20.6(3.9)$ & $22.3(3.0)$ & $F_{2,69}=3.04, p=0.054$ \\
\hline Education $\left(\right.$ year) $^{\mathrm{b}}$ & $13.4(2.5)$ & $13.1(2.6)$ & $14.5(1.6)$ & $F_{2,69}=2.29, p=0.109$ \\
\hline Premorbid $\mathrm{IQ}^{\mathrm{b}}$ & $104(9.0)$ & $105(8.1)$ & $110(6.4)$ & $F_{2,69}=3.03, p=0.055$ \\
\hline DOI (weeks) & $46.5(47.0)$ & & & \\
\hline PANSS $^{\mathrm{C}}$ total & $68.9(19.6)$ & $67.7(14.3)$ & & $t_{46}=0.238, p=0.813$ \\
\hline Positive & $15.2(5.3)$ & $14.3(3.7)$ & & $t_{46}=0.684, p=0.497$ \\
\hline Negative & $18.8(7.0)$ & $17.3(4.7)$ & & $t_{46}=0.874, p=0.387$ \\
\hline General & $34.9(10.0)$ & $36.1(8.0)$ & & $t_{46}=-0.466, p=0.643$ \\
\hline GAF score ${ }^{c}$ & $38(11)$ & $48(10)$ & & $t_{46}=-3.35, p \equiv \underline{0.002}$ \\
\hline Antipsychotic dose $(\mathrm{mg} / \text { day })^{c}$ & $388(284)$ & $174(262)$ & & $t_{46}=2.70, p=0.010$ \\
\hline
\end{tabular}

All values are shown as mean (standard deviation) except for the first row. Underlined results indicate $p<0.05$

ROSZ recent-onset schizophrenia, UHR ultra-high risk, $H C$ healthy control, IQ intelligence quotient, DOI duration of illness, PANSS positive and negative symptom scale, GAF global assessment of functioning, ANOVA analysis of variance, $d f$ degrees of freedom

${ }^{a}$ Chi-square test

bOne-way ANOVA

Independent $t$-test

Both glutamatergic dysfunction and GABAergic dysfunction may reflect an altered excitatory/inhibitory (E/I) balance in schizophrenia. Excitation is mediated by pyramidal neurons that release glutamate as neurotransmitters, and inhibition is mediated by interneurons that release GABA as neurotransmitters. Because pyramidal neurons and interneurons are connected with each other and make networks ${ }^{14}$, glutamatergic excitation and GABAergic inhibition affect each other and contribute to the E/I balance ${ }^{15}$. Recent animal studies have shown that interactions between abnormalities in NMDAR and dysfunction of GABAergic interneurons lead to alterations in E/I balance. Repeated administration of NMDAR antagonists $^{16-20}$ and genetic deletion of NMDAR ${ }^{21-23}$ cause dysfunction of GABAergic interneurons that cause disinhibition of pyramidal neurons and lead to alterations in E/I balance. Decreased expression of the GABA synthetic enzyme GAD67 causes alterations in NMDARdependent processes and leads to alterations in E/I balance $^{24,25}$. Therefore, glutamatergic dysfunction and GABAergic dysfunction in schizophrenia may interact with each other and lead to alterations in E/I balance.

The mismatch negativity (MMN), an electrophysiological index of auditory deviance detection, is expected to be a useful biomarker for NMDAR dysfunction in schizophrenia ${ }^{26-29}$, because NMDAR antagonists reduce MMN amplitude ${ }^{30-32}$. Many original investigations and subsequent meta-analyses have demonstrated MMN amplitude reduction in individuals with chronic schizophrenia $^{33-38}$, first-episode psychosis ${ }^{38-43}$, and ultrahigh risk for psychosis (UHR) ${ }^{38,42,44-49}$. Some previous studies have shown that MMN amplitude reduction predicts the onset of psychosis in individuals with $\mathrm{UHR}^{47-49}$. In those studies, the MMN in converters showed significantly greater amplitude reduction than that in nonconverters. Furthermore, previous investigations have shown that in schizophrenia patients, MMN is correlated with the plasma level of glutamate ${ }^{50}$, which is significantly correlated with CSF levels of glutamate ${ }^{51,52}$, and with the level of glutamate in the ACC measured using ${ }^{1} \mathrm{H}-\mathrm{MRS}^{53}$.

In contrast, gamma-band auditory steady-state response (ASSR) is a candidate electrophysiological index of GABAergic abnormalities in the auditory cortex in schizophrenia. Cortical gamma oscillations are evoked by synaptic interactions between PV-positive GABAergic interneurons and pyramidal neurons ${ }^{54,55}$. Previous studies have reported deficits of gamma-band ASSR in chronic schizophrenia ${ }^{56-62}$ and first-episode psychosis ${ }^{63-65}$. For UHR, Tada et al. ${ }^{65}$ showed a specific time-course ASSR alteration, with reduction in the late-latency component, while leaving early-latency component intact. Recent meta-analysis confirmed impairment of the $40 \mathrm{~Hz}$ ASSR in patients with schizophrenia ${ }^{66}$.

Accordingly, we hypothesize that there is an abnormal association between NMDAR and GABAergic dysfunction surrounding the auditory cortex, which may lead to altered E/I balance at around the onset of schizophrenia. However, there is a lack of in vivo electrophysiological evidence of an association between NMDAR function and GABA function in individuals in the early stages of psychosis. In this study, we used MMN and gamma-band ASSR as sensitive electrophysiological markers of 
NMDAR and GABAergic interneurons, respectively. We predicted that impaired MMN and reduced gamma-band ASSR would show a correlation around the onset of schizophrenia.

\section{Materials and methods Subjects}

We recorded electroencephalograms (EEGs) of 21 individuals with recent-onset schizophrenia (ROSZ), 27 individuals with UHR, and 24 healthy controls (HCs; Table 1). Fourteen of the participants had also participated in our previous MMN study ${ }^{42}$, and 38 had participated in our previous ASSR study ${ }^{65}$. Individuals with ROSZ or UHR were recruited from outpatient and inpatient units at the University of Tokyo Hospital. The HC group was recruited through advertisements at several universities in Tokyo. Inclusion criteria for ROSZ individuals were that they were diagnosed using the Diagnostic and Statistical Manual of Mental Disorders, Fourth Edition (DSM-IV), aged 15-40 years, and had onset of continuous psychotic symptoms within the past 60 months. Among a total of 21 ROSZ patient sample, $N=15$ underwent EEG measurement as the biomarker assessments at Time 1 for the Integrative Neuroimaging Studies for Schizophrenia Targeting Early Intervention and Prevention (IN-STEP) project ${ }^{67}$ [all satisfied the criteria of first-episode schizophrenia (FES): continuous psychotic symptoms within the past 60 months and no history of antipsychotic drug treatment for more than 16 cumulative weeks at entry into the IN-STEP project]; $N=1$ did not undergo EEG testing at Time 1, but instead underwent the initial EEG testing during the follow-up period of INSTEP; and $N=5$ were newly recruited ( $N=3$ satisfied FES). Inclusion criteria for individuals with UHR were identified using the Structured Interview for Prodromal Symptoms $(\mathrm{SIPS})^{68}$, and all were aged $15-30$ years. Inclusion criteria of $\mathrm{HC}$ were that they were aged 15-40 years and had no personal history of psychiatric disease or a family history of axis I disorders in first-degree relatives. Exclusion criteria for all groups were neurological illness, traumatic brain injury with loss of consciousness for more than $5 \mathrm{~min}$, history of electroconvulsive therapy, low premorbid intelligence quotient (IQ; below 70), previous alcohol/substance abuse or addiction, or a hearing impairment, which was assessed with a hearing test in both ears at $30-\mathrm{dB}$ sound pressure level tone at $1000 \mathrm{~Hz}$ and $40-\mathrm{dB}$ at $4000 \mathrm{~Hz}$ by audiometer. Written informed consent was obtained from each subject before participation. The Research Ethics Committee of the Faculty of Medicine at the University of Tokyo approved this study (approval No. 629 and 2226).

The estimated premorbid IQ was assessed using the Japanese version of the National Adult Reading Test in all participants ${ }^{69,70}$. The Positive and Negative Syndrome
Scale (PANSS) ${ }^{71}$ and Global Assessment of Functioning $(\mathrm{GAF})^{72,73}$ were used for assessment of global clinical symptoms and functioning in all participants with ROSZ or UHR. Nineteen patients with ROSZ and 17 individuals with UHR took antipsychotic medication. The antipsychotic dose was converted to an equivalent dose of chlorpromazine ${ }^{74}$.

\section{Procedure and analyses of the mismatch negativity}

A two-tone auditory oddball paradigm with 2000 stimuli was used for MMN. Standard tones $(1000 \mathrm{~Hz}, 50 \mathrm{~ms})$ were $90 \%$ of the stimuli and duration-deviant tones $(1000 \mathrm{~Hz}, 100 \mathrm{~ms})$ were $10 \%$ of the stimuli. All stimuli were $80 \mathrm{~dB}$ and had $1 \mathrm{~ms}$ rise/fall time. Stimulus onset asynchrony was $500 \mathrm{~ms}$. While the participants watched a silent cartoon, the tones were presented binaurally through inserted earphones (Multi Trigger System, Medical Try System, Tokyo, Japan).

We used a 64-channel Geodesic EEG System (Electrical Geodesics Inc., Eugene, OR) to obtain EEG data. Electrodes were referenced to the vertex, and impedances were maintained below $50 \mathrm{k} \Omega$. The sampling rate was $500 \mathrm{~Hz}$. The analog filter bandpass was set at $0.1-100 \mathrm{~Hz}$. We analyzed EEG data using EEGLAB ${ }^{75}$. The continuous EEG data were re-referenced to an average reference, digitally filtered at $0.1-20 \mathrm{~Hz}$, and segmented from -100 to $500 \mathrm{~ms}$ relative to the stimulus onset. The mean of the pre-stimulus baseline was subtracted for baseline correction. Independent component analysis was used for eye blink correction. Epochs exceeding $\pm 100 \mu \mathrm{V}$ at any electrode were rejected. After averaging across trials, the event-related potential (ERP) waveform in response to standard stimuli was subtracted from the ERP waveform in response to deviant stimuli.

The amplitude of MMN at seven electrodes around the $\mathrm{FCz}$ was used for MMN analysis because the largest MMN amplitudes were obtained with them (Supplementary Fig. 1). The MMN amplitude was measured using the mean voltage from 135 to 205 ms post stimuli, in accordance with previous studies ${ }^{36,42,76}$.

We performed another oddball paradigm with response to frequency deviants, and the oddball paradigms were counterbalanced. However, we did not employ the frequency-deviant MMN in subsequent analyses because the group difference of MMN was specific to the duration-deviant MMN.

\section{Procedure and analyses of the auditory steady-state response}

The ASSR paradigm used in this study, which is described in detail elsewhere ${ }^{65}$, was similar to those used in previous studies from different laboratories ${ }^{56,58}$. Briefly, subjects were instructed to relax with their eyes open, and they received auditory stimuli presented binaurally 
through inserted earphones, the same as those used in the MMN session. We performed the ASSR session before the MMN session, within $30 \mathrm{~min}$. We recorded ASSR at first to avoid muscle artifacts because gamma band ASSR is sensitive to muscle artifacts ${ }^{77}$. We measured both ASSR and MMN in total within $60 \mathrm{~min}$. Click sounds $(80 \mathrm{~dB}$, $1 \mathrm{~ms}$ ) presented in $500 \mathrm{~ms}$ trains at 20,30 , and $40 \mathrm{~Hz}$ served as the auditory stimuli. Click sound trains were 200 trains at each frequency. The intertrain interval was $500 \mathrm{~ms}$. The sampling rate was $250 \mathrm{~Hz}$. The analog filter bandpass was set at $0.1-100 \mathrm{~Hz}$. We used $40 \mathrm{~Hz}$ ASSR data for subsequent analyses because impairment of $40 \mathrm{~Hz}$ ASSR in early stages of schizophrenia was confirmed in previous studies ${ }^{63-65}$.

We analyzed EEG data by using EEGLAB ${ }^{75}$. The continuous EEG data were re-referenced to an average reference, a high-pass filter $(1 \mathrm{~Hz})$ and a notch filter $(50 \mathrm{~Hz})$ were applied to them in order to remove artifacts, and they were segmented from -250 to $750 \mathrm{~ms}$, relative to the stimulus onset. Independent component analysis was used for eye blink correction, and epochs exceeding \pm $100 \mu \mathrm{V}$ at any electrode were rejected.

We performed time-frequency analyses with a shortterm Fourier transformation and then calculated intertrial phase coherence (ITC) and event-related spectral perturbation (ERSP) as indices of ASSR. The ITC indicates phase consistency across trials and ranges between 0 (random phase across trials) and 1 (identical phase across trials). The ERSP indicates event-related changes in power relative to a pre-stimulus baseline. Decreases in ITC and ERSP reflect reduced neural responses to auditory steadystate stimulation. We calculated the mean ITC and ERSP by averaging the data over stimulation time $(0-500 \mathrm{~ms})$ and stimulation frequency $(40 \mathrm{~Hz}: 36-45 \mathrm{~Hz})$. Because our previous study ${ }^{65}$ observed differential alteration of early- and late-time-course components of ITC and ERSP in early stages of psychosis, we additionally calculated the mean ITC and ERSP for each 100 ms-epoch for timecourse analyses. We focused on the frontocentral electrode site $(\mathrm{FCz})$ because the most prominent ASSR was found at FCz.

\section{Statistical analyses}

We used SPSS (Version 23.0.0.0, IBM Corp., Armonk, NY, USA) for all statistical analyses. We employed $\chi^{2}$ tests, independent $t$-tests, and one-way ANOVAs for comparison of demographics and clinical characteristics among the groups. We performed one-way ANOVAs for comparison of the number of epochs and found no significant differences among groups [MMN, $161 \pm 30$ for ROSZ, $156 \pm 33$ for UHR, and $164 \pm 24$ for HCs $\left(F_{2}, 69=\right.$ $0.44, p=0.65)$; ASSR, $182 \pm 21$ for ROSZ, $183 \pm 16$ for $\mathrm{UHR}$, and $187 \pm 10$ for $\left.\mathrm{HCs}\left(F_{2,69}=0.62, p=0.54\right)\right]$. For the main comparison of MMN amplitude, we used repeated measures ANOVA with the three groups as the between-subject factors and with seven electrodes around the $\mathrm{FCz}$ as the within-subject factor. For the main comparison of ITC (0-500 ms) and ERSP $(0-500 \mathrm{~ms})$, we used one-way ANOVA. As a supplementary analysis, we performed repeated measures ANOVA with the three groups as the between-subjects factor and with time blocks $(0-100,100-200,200-300,400-500 \mathrm{~ms})$ as the withinsubject factor. Greenhouse-Geisser correction was used for repeated measure ANOVAs when appropriate. In case of a significant group-by-time interaction, we used oneway ANOVA and post-hoc tests with Bonferroni correction for comparison of ITC and ERSP time blocks, and Cohen's $d$ effect sizes were calculated for group comparisons of MMN, ITC, and ERSP. The significance level was set at $p<0.05$ (two-tailed).

The Pearson correlation coefficients $(r)$ of MMN with ITC and ERSP in each group were calculated. $p<0.0014$ $(0.05 / 36)$ was considered statistically significant based on Bonferroni correction (two-tailed). Additionally, we performed correlation analyses between frequency-deviant MMN and ASSR [20,30, and $40 \mathrm{~Hz}$; ITC $(0-500 \mathrm{~ms})$ and ERSP $(0-500 \mathrm{~ms}) ; p<0.0028(0.05 / 18)$ was considered statistically significant based on Bonferroni correction (two-tailed)] and between the duration-deviant MMN and ASSR $[20$ and $30 \mathrm{~Hz}$; ITC $(0-500 \mathrm{~ms})$ and ERSP $(0-500 \mathrm{~ms}) ; p<0.0042(0.05 / 12)$ was considered statistically significant based on Bonferroni correction (twotailed)] in ROSZ, UHR and HCs for supplementary information (Supplementary Table 1 and 2, and Supplementary Text).

If we found a significant correlation of MMN with ITC and ERSP in the group, we further tested whether the correlation was specific to the group by comparing Fisher's $r$-to- $z$ transformed correlational coefficients between the groups. Potential effects of age and premorbid IQ on MMN and ITC were tested with correlation analyses between age and MMN/ITC, and between premorbid IQ and MMN/ITC in ROSZ because age $\left(F_{2}, 69=3.04, p=\right.$ 0.054; Table 1$)$ and premorbid IQ $\left(F_{2,69}=3.03, p=0.055\right.$; Table 1) were different among the three groups (ROSZ, UHR, and HC) at the trend level. Furthermore, we performed partial correlation analyses between MMN and ITC, after adjustment for age and premorbid IQ in ROSZ. In addition, we sought to parse out the effects of medication on our main findings. We first calculated Pearson's $r$ between the antipsychotic dose (chlorpromazine equivalents) and MMN/ITC in ROSZ. The partial correlation to adjust for medication effects was calculated for the combination of the MMN amplitude and ITC in ROSZ. The significance level was set at $p<0.05$ (twotailed). 


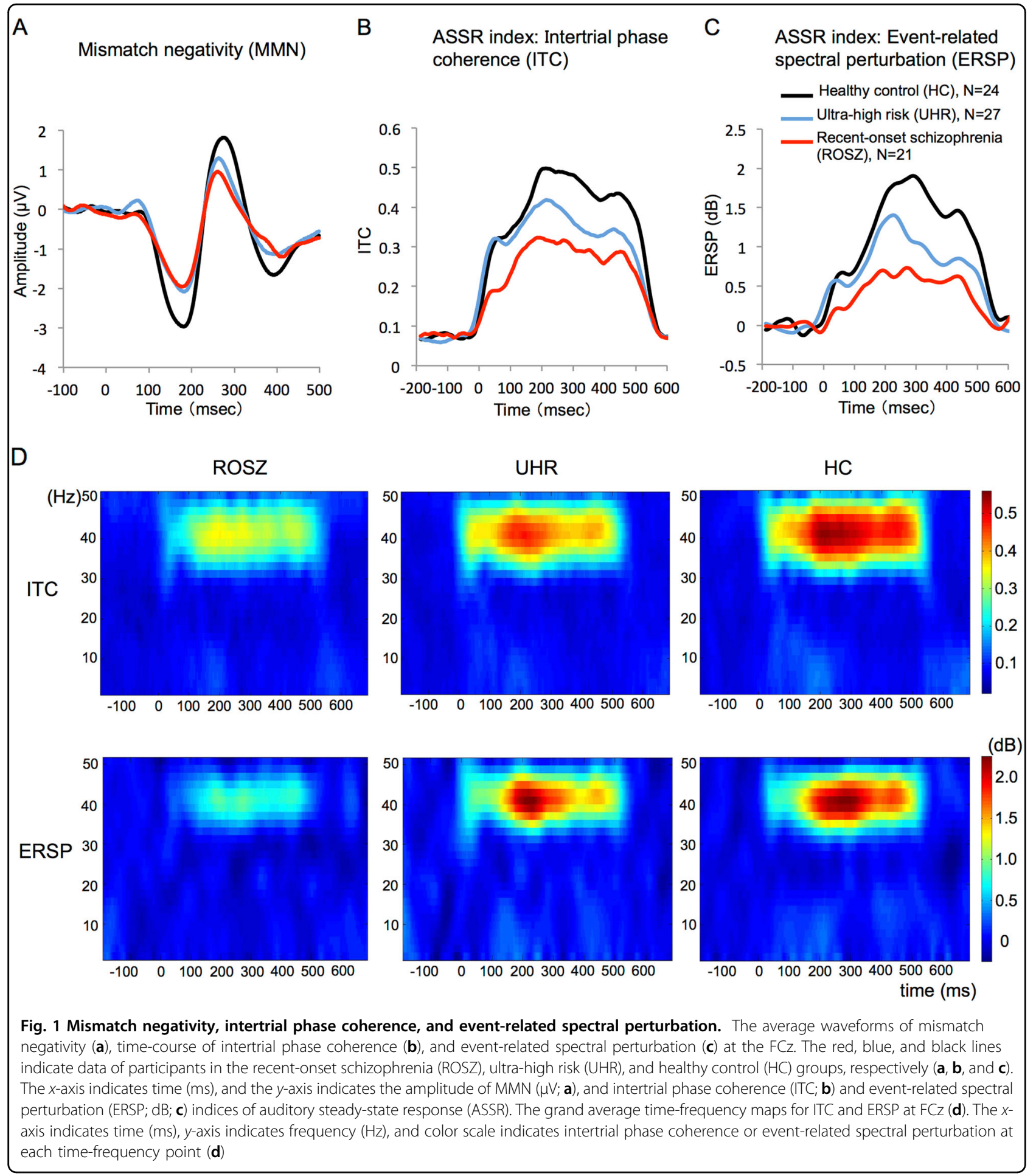

\section{Results}

\section{Mismatch negativity}

The average waveforms for MMN in the ROSZ, UHR, and $\mathrm{HC}$ groups are shown in Fig. 1a. The mean amplitude (SD) of MMN was $-1.48(0.80)$ in the ROSZ group, -1.61 (0.84) in the UHR group, and $-2.44(0.81)$ in the HC group. Repeated measures ANOVA revealed a significant main effect of group $\left(F_{2,67}=8.82, p<0.001\right)$ and a significant main effect of electrode $\left(F_{4,268}=10.9, p<0.001\right)$ but no significant group-by-electrode interaction $\left(F_{8,268}\right.$ $=0.29, p=0.97)$. The post-hoc tests with Bonferroni correction indicated that the MMN amplitude was 
Table 2 Group comparison of ASSR indices (ITC and ERSP)

\begin{tabular}{|c|c|c|c|c|c|c|c|c|c|c|c|c|c|}
\hline & \multicolumn{7}{|c|}{ One-way ANOVA } & \multicolumn{6}{|c|}{ Post-hoc analysis ${ }^{a}$} \\
\hline & \multicolumn{2}{|l|}{ ROSZ } & \multicolumn{2}{|l|}{ UHR } & \multicolumn{2}{|l|}{$\mathrm{HC}$} & \multirow[t]{2}{*}{ Statistics } & \multicolumn{2}{|c|}{ ROSZ-UHR } & \multicolumn{2}{|c|}{ ROSZ-HC } & \multicolumn{2}{|c|}{ UHR-HC } \\
\hline & Mean & SD & Mean & SD & Mean & SD & & $p^{\mathrm{a}}$ & $d$ & $p^{\mathrm{a}}$ & $d$ & $p^{\mathrm{a}}$ & $d$ \\
\hline \multicolumn{14}{|c|}{ ITC [0-1, arbitrary unit] } \\
\hline $0-500 \mathrm{~ms}$ & 0.27 & 0.12 & 0.35 & 0.11 & 0.41 & 0.11 & $F_{2,69}=9.29, p \leq \underline{0.001}$ & $\underline{0.032}$ & -0.75 & $\leq \underline{0.001}$ & -1.27 & 0.202 & -0.53 \\
\hline \multicolumn{14}{|l|}{ Time blocks } \\
\hline $0-100 \mathrm{~ms}$ & 0.18 & 0.07 & 0.29 & 0.09 & 0.28 & 0.09 & $F_{2,69}=12.6, p \leq \underline{0.001}$ & $\leq 0.001$ & -1.46 & 0.001 & -1.23 & 1.000 & 0.20 \\
\hline $100-200 \mathrm{~ms}$ & 0.29 & 0.12 & 0.38 & 0.13 & 0.41 & 0.10 & $F_{2,69}=5.86, p \equiv \underline{0.004}$ & $\underline{0.037}$ & -0.70 & $\underline{0.005}$ & -1.05 & 1.000 & -0.24 \\
\hline $200-300 \mathrm{~ms}$ & 0.31 & 0.16 & 0.41 & 0.14 & 0.49 & 0.13 & $F_{2,69}=8.66, p \leq \underline{0.001}$ & 0.069 & -0.64 & $\leq \underline{0.001}$ & -1.24 & 0.142 & -0.60 \\
\hline $300-400 \mathrm{~ms}$ & 0.28 & 0.13 & 0.34 & 0.12 & 0.45 & 0.14 & $F_{2,69}=9.27, p \leq \underline{0.001}$ & 0.337 & -0.48 & $\leq \underline{0.001}$ & -1.22 & $\underline{0.018}$ & -0.79 \\
\hline $400-500 \mathrm{~ms}$ & 0.27 & 0.13 & 0.33 & 0.12 & 0.42 & 0.13 & $F_{2,69}=8.32, p \equiv \underline{0.001}$ & 0.291 & -0.50 & $\leq \underline{0.001}$ & -1.17 & $\underline{0.039}$ & -0.72 \\
\hline \multicolumn{14}{|l|}{ ERSP $[\mathrm{dB}]$} \\
\hline $0-500 \mathrm{~ms}$ & 0.49 & 0.64 & 0.88 & 0.57 & 1.30 & 0.69 & $F_{2,69}=9.18, p \leq \underline{0.001}$ & 0.118 & -0.64 & $\leq \underline{0.001}$ & -1.21 & 0.061 & -0.67 \\
\hline \multicolumn{14}{|l|}{ Time blocks } \\
\hline 0-100 ms & 0.17 & 0.41 & 0.51 & 0.45 & 0.50 & 0.41 & $F_{2,69}=4.47, p \equiv \underline{0.015}$ & 0.026 & -0.78 & 0.040 & -0.79 & 1.000 & 0.03 \\
\hline $100-200 \mathrm{~ms}$ & 0.55 & 0.66 & 0.92 & 0.61 & 1.15 & 0.65 & $F_{2,69}=4.99, p \equiv \underline{0.009}$ & 0.145 & -0.59 & $\underline{0.007}$ & -0.91 & 0.637 & -0.36 \\
\hline 200-300 ms & 0.67 & 0.90 & 1.25 & 0.88 & 1.86 & 0.94 & $F_{2,69}=9.67, p \leq \underline{0.001}$ & 0.090 & -0.65 & $\leq 0.001$ & -1.29 & 0.060 & -0.67 \\
\hline $300-400 \mathrm{~ms}$ & 0.57 & 0.83 & 0.89 & 0.71 & 1.62 & 0.93 & $F_{2,69}=9.84, p \leq \underline{0.001}$ & 0.549 & -0.42 & $\leq \underline{0.001}$ & -1.19 & $\underline{0.007}$ & -0.88 \\
\hline $400-500 \mathrm{~ms}$ & 0.50 & 0.78 & 0.80 & 0.56 & 1.36 & 0.86 & $F_{2,69}=8.13, p \equiv \underline{0.001}$ & 0.508 & -0.44 & 0.001 & -1.05 & $\underline{0.023}$ & -0.78 \\
\hline
\end{tabular}

$d$ means Cohen's $d$ effect size. Underlined results indicate $p<0.05$

ANOVA analysis of variance, SD standard deviation, ROSZ recent-onset schizophrenia, UHR ultra-high risk, HC healthy control, ASSR auditory steady-state response, ITC intertrial phase coherence, ERSP event-related spectral perturbation

${ }^{a}$ Bonferroni correction for three contrasts (ROSZ-UHR, ROSZ-HC, UHR-HC)

significantly smaller in the ROSZ group $(p=0.001, d=$ $1.20)$ and the UHR group ( $p=0.003, d=1.01)$ than in the $\mathrm{HC}$ group. There were no significant differences in MMN between ROSZ and UHR ( $p=1.00, d=0.16)$. Because we found no significant group-by-electrode interaction, we used the mean MMN amplitude at seven electrodes for further analyses.

\section{Auditory steady-state response}

The time-courses of the ITC or ERSP, which are indices of ASSR, are shown in Fig. 1b, c. The grand average timefrequency maps for ITC or ERSP are shown in Fig. 1d. For the analysis of overall ITC $(0-500 \mathrm{~ms})$, there was a significant main effect of group $\left(F_{2}, 69=9.29, p<0.001\right.$; Table 2). The post-hoc tests showed that ITC (0-500 ms) was significantly smaller in ROSZ than in UHR $(p=0.032$, $d=-0.75)$ and HCs $(p<0.001, d=-1.27)$. There were no significant differences in ITC $(0-500 \mathrm{~ms})$ between UHR and HCs $(p=0.20, d=-0.53)$. For additional analysis of time-course of ITC, repeated measures ANOVA showed a significant group-by-time interaction $\left(F_{5,161}=\right.$ $5.41, p<0.001)$. Subsequently, the post-hoc tests showed a significant difference between ROSZ and HCs in all five, between ROSZ and UHR in early latency (0-100, 100-200 ms), and between UHR and HCs in late latency (300-400, 400-500 ms) time blocks.

For the analysis of overall ERSP $(0-500 \mathrm{~ms})$, there was a significant main effect of group $\left(F_{2,69}=9.18, p<0.001\right.$; Table 2). The post-hoc tests showed that ERSP (0-500 ms) was significantly smaller in ROSZ than in HCs $(p<0.001, d=-1.21)$. There were no significant differences in ERSP (0-500 ms) between ROSZ and UHR ( $p=$ $0.12, d=-0.64)$ or between UHR and HCs $(p=0.061, d$ $=-0.67$ ). For additional analysis of the time-course of ERSP, a repeated measures ANOVA showed a significant group-by-time interaction $\left(F_{4,151}=5.66, p<0.001\right)$. The post-hoc tests showed a significant difference between ROSZ and HCs in all five, between ROSZ and UHR in early latency (0-100 ms), and between UHR and HCs in late latency (300-400, 400-500 ms) time blocks. 
Table 3 Correlation of MMN amplitude with ASSR indices (ITC and ERSP)

\begin{tabular}{|c|c|c|c|c|c|c|}
\hline & \multicolumn{6}{|l|}{ MMN } \\
\hline & \multicolumn{2}{|l|}{ ROSZ } & \multicolumn{2}{|l|}{ UHR } & \multicolumn{2}{|l|}{$\mathrm{HC}$} \\
\hline & $r$ & $p$ & $r$ & $p$ & $r$ & $p$ \\
\hline \multicolumn{7}{|l|}{ ITC } \\
\hline $0-500 \mathrm{~ms}$ & -0.69 & $\leq \underline{0.001}$ & -0.25 & 0.20 & -0.26 & 0.22 \\
\hline \multicolumn{7}{|l|}{ Time blocks } \\
\hline 0-100 ms & -0.13 & 0.56 & -0.04 & 0.86 & -0.29 & 0.17 \\
\hline $100-200 \mathrm{~ms}$ & -0.33 & 0.14 & -0.26 & 0.19 & -0.13 & 0.53 \\
\hline $200-300 \mathrm{~ms}$ & -0.29 & 0.21 & -0.33 & 0.10 & -0.16 & 0.46 \\
\hline $300-400 \mathrm{~ms}$ & -0.36 & 0.11 & -0.27 & 0.17 & -0.29 & 0.17 \\
\hline $400-500 \mathrm{~ms}$ & -0.37 & 0.10 & -0.18 & 0.38 & -0.30 & 0.15 \\
\hline \multicolumn{7}{|l|}{ ERSP } \\
\hline $0-500 \mathrm{~ms}$ & -0.17 & 0.47 & -0.31 & 0.11 & -0.29 & 0.16 \\
\hline \multicolumn{7}{|l|}{ Time blocks } \\
\hline $0-100 \mathrm{~ms}$ & 0.09 & 0.69 & -0.19 & 0.34 & -0.39 & 0.06 \\
\hline $100-200 \mathrm{~ms}$ & -0.07 & 0.75 & -0.17 & 0.40 & -0.22 & 0.31 \\
\hline $200-300 \mathrm{~ms}$ & -0.14 & 0.55 & -0.32 & 0.10 & -0.09 & 0.68 \\
\hline $300-400 \mathrm{~ms}$ & -0.21 & 0.35 & -0.36 & 0.07 & -0.29 & 0.17 \\
\hline $400-500 \mathrm{~ms}$ & -0.28 & 0.22 & -0.29 & 0.14 & -0.41 & 0.05 \\
\hline
\end{tabular}

Underlined result indicates $p<0.0014(0.05 / 36)$

ROSZ recent-onset schizophrenia, UHR ultra-high risk, HC healthy control, MMN mismatch negativity, ASSR auditory steady-state response, ITC intertrial phase coherence, ERSP event-related spectral perturbation

\section{Correlations between MMN amplitude and ASSR indices (ITC and ERSP)}

The MMN amplitude was significantly correlated with overall ITC $(0-500 \mathrm{~ms})$ in ROSZ $(r=-0.69, p<0.001$; Table 3 and Fig. 2). However, the correlation between MMN amplitude and ITC (0-500 ms) was not significant in UHR $(r=-0.25, p=0.20)$ or HCs $(r=-0.26, p=$ 0.22 ). The MMN amplitude was not significantly correlated with the overall ERSP $(0-500 \mathrm{~ms})$ for either group. Additionally, the MMN amplitude was not significantly correlated with ITC or ERSP for any time-course block.

The correlation between MMN and ITC was specific to the ROSZ group compared to the UHR group $(z=1.92, p$ $=0.055)$ and the HC group $(z=1.83, p=0.067)$ at the trend level. Further, we evaluated potential effects of age and premorbid IQ on MMN and ITC with the correlation analyses between age and MMN/ITC, and between premorbid IQ and MMN/ITC in ROSZ. Age was not significantly correlated with MMN $(r=0.11, p=0.64)$ or ITC $(r=-0.23, p=0.33)$ in ROSZ. Premorbid IQ was not significantly correlated with MMN $(r=-0.22, p=0.34)$ or ITC $(r=0.25, p=0.28)$ in ROSZ. In addition, partial correlation analyses between MMN and ITC after adjustment for age $(r=-0.69, p=0.001)$ and premorbid IQ $(r=-0.68, p=0.001)$ showed almost the same results compared with the original results in ROSZ. An antipsychotic dose was not significantly correlated with MMN $(r=0.29, p=0.21)$ or ITC $(0-500 \mathrm{~ms} ; r=-0.42, p=$ $0.061)$ in ROSZ. A partial correlational analysis after adjustment for the antipsychotic dose between MMN and ITC $(0-500 \mathrm{~ms})$ in ROSZ was performed, and the correlation remained significant $(r=-0.66, p=0.002)$.

\section{Discussion}

We obtained the following results: (i) the MMN amplitude was reduced in ROSZ and UHR; (ii) ITC and ERSP were significantly reduced in ROSZ, and earlylatency ITC and ERSP were intact, while late-latency ITC and ERSP were impaired in UHR; and (iii) as the main finding, the MMN amplitude was significantly correlated with the ITC in ROSZ, but not in UHR. This study is the first to identify an association between electrophysiological indices of glutamate and GABA function in vivo in early psychosis.

Our results of reduced MMN both in ROSZ and UHR are consistent with those of previous studies on early stages of psychosis ${ }^{39-47}$. These findings indicate that NMDAR shows dysfunction before the onset of schizophrenia. The overall indices of gamma-band ASSR (ITC and ERSP) are also reduced in recent-onset schizophrenia, consistent with previous findings ${ }^{63,65}$. Confirming our previous investigation ${ }^{65}$, in UHR, early-latency ITC and ERSP were intact, while late-latency ITC and ERSP were impaired. These patterns of abnormality suggest that GABAergic interneuron dysfunction may, at least in part, develop through the onset of psychosis, which might explain the observation that an abnormal association of MMN and ASSR is evident in ROSZ alone.

The main finding of our study was a significant correlation between the MMN amplitude and gamma-band ASSR in ROSZ, but not in UHR. The correlation was specific to the ROSZ group compared to the UHR group at the trend level. This may reflect altered $\mathrm{E} / \mathrm{I}$ balance in ROSZ but not in UHR. Previous animal studies have shown that NMDAR hypofunction causes dysfunction of GABAergic interneurons ${ }^{21-23}$. Belforte et $\mathrm{al}^{21}$. found that elimination of the NR1 subunit of the NMDAR in cortical and hippocampal interneurons caused a reduction in the expression of GAD67 and PV. Carlen et al. ${ }^{23}$ reported that NMDAR dysfunction in PV interneurons caused impairments in gamma oscillations. Furthermore, Nakazawa et $\mathrm{al}^{78}$ described that the first NMDAR hypofunction occurs in PV-positive GABA interneurons, in early postnatal development, which would impair the cortical maturation that causes a reduction in intrinsic excitability 

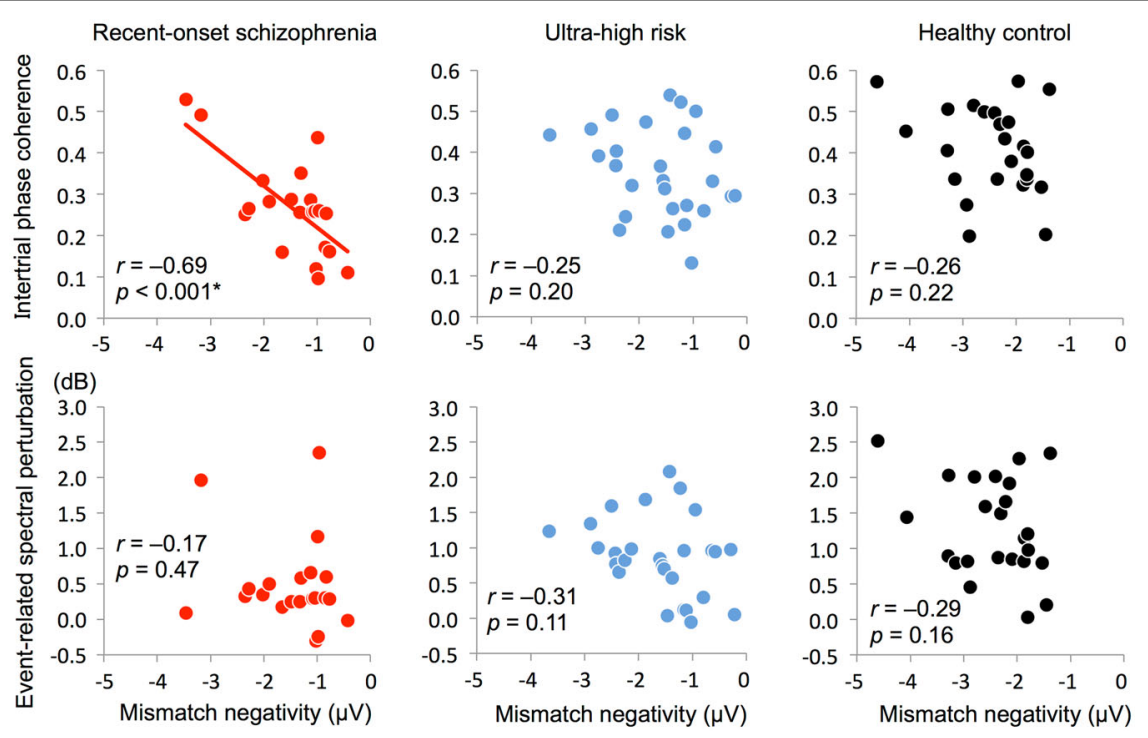

Fig. 2 Correlation of mismatch negativity with intertrial phase coherence or event-related spectral perturbation. The $x$-axis indicates mismatch negativity $(\mu \mathrm{V})$, and the $y$-axis indicates intertrial phase coherence or event-related spectral perturbation (dB). The asterisk indicates $p<$ $0.0014(0.05 / 36)$

and impaired GABA release, thus leading to the disinhibition of pyramidal neurons. Altogether, these findings suggest that the dysfunction of NMDARs cause dysfunction of GABA interneurons that cause disinhibition of pyramidal neurons and leads to alterations in E/I balance. Decreased expression of the GABA synthetic enzyme GAD67 causes alterations in NMDAR-dependent processes and lead to alterations in E/I balance ${ }^{24,25}$. Therefore, glutamatergic dysfunction and GABAergic dysfunction may interact with each other and lead to altered $\mathrm{E} / \mathrm{I}$ balance in ROSZ. On the other hand, individuals with UHR showed reduced MMN and ASSR, but there were no significant correlations between MMN and ASSR. These findings suggest that individuals with UHR may have glutamatergic dysfunction and GABAergic dysfunction but that interactions between glutamatergic dysfunction and GABAergic dysfunction may not be strong enough to lead to an altered E/I balance in UHR. Therefore, glutamatergic dysfunction and GABAergic dysfunction may indicate a risk for psychosis, and interactions between glutamatergic dysfunction and GABAergic dysfunction and subsequent alterations in E/I balance may lead to the onset of psychosis. In contrast to ITC, ERSP showed no significant correlation with MMN. Previous animal studies have shown that dysfunction of NMDAR on GABAergic interneuron decrease evoked power of gamma oscillations but increase spontaneous power of gamma oscillations ${ }^{23,79}$. Because ERSP reflects power of gamma oscillations and includes both evoked power and spontaneous power, the mixed effects of decreased evoked power and increased spontaneous power may obscure the association between MMN and gamma oscillations. Because ITC does not reflect the power of gamma oscillations, the mixed effects of power do not affect the association.

The study has some limitations. First, potential medication effects might have influenced our findings. However, our main findings remained significant when we applied a partial correlation analysis to adjust for medication effects. Second, this study was a cross-sectional study. Future longitudinal studies will be required to clarify the longitudinal course of the association between MMN amplitude and gamma-band ASSR around the onset of psychosis.

In conclusion, our observations provide the first in vivo electrophysiological evidence that an abnormal association of NMDAR-GABA dysfunctions presumably surrounding the auditory cortex may be present in recentonset schizophrenia. The MMN and gamma-band ASSR may be useful sensitive markers in the development of early intervention strategies for psychosis, in order to target the alleviation of this aberrant association.

\section{Acknowledgements}

We thank the members of the Integrative Neuroimaging Studies for Schizophrenia Targeting Early Intervention and Prevention (IN-STEP) research team in the University of Tokyo Hospital for their advice and assistance with this study. We gratefully acknowledge all the participants of this study. This study was supported by JSPS KAKENHI Grant Numbers JP16H06395, 16H06399, 16 K21720 (K.Ka), 15K19713 (K.Ki.), 15 H06166 (M.T.), and 15K09858 (M.S.), by the Brain Mapping by Integrated Neurotechnologies for Disease Studies (Brain/ MINDS) from the Japan Agency for Medical Research and Development, AMED (JP17dm0207004; K. Ka), by the SENSHIN Medical Research Foundation (M.T.), by the Takeda Science Foundation (M.T), by UTokyo Center for Integrative Science of Human Behavior (CiSHuB; S.K., K.Ka), and by the International 
Research Center for Neurointelligence (WPI-IRCN) at the University of Tokyo Institutes for Advanced Study (UTIAS; M.T., S.K., \& K.Ka). The funders played no role in the study design, data collection and analysis, publication decision, or manuscript preparation.

\section{Author details}

'Department of Neuropsychiatry, Graduate School of Medicine, The University of Tokyo, 7-3-1, Hongo, Bunkyo-ku, Tokyo 113-8655, Japan. ${ }^{2}$ The International Research Center for Neurointelligence (WPI-IRCN) at The University of Tokyo Institutes for Advanced Study (UTIAS), The University of Tokyo, 7-3-1, Hongo, Bunkyo-ku, Tokyo 113-8655, Japan. ${ }^{3}$ Department of Psychiatry, Kawamuro Memorial Hospital, 71, Kitashinbo, Joetsu-shi, Niigata 943-0109, Japan. ${ }^{4}$ University of Tokyo Institute for Diversity \& Adaptation of Human Mind (UTIDAHM), 3-8-1, Komaba, Meguro-ku, Tokyo 153-8902, Japan. ${ }^{5}$ Center for Evolutionary Cognitive Sciences, Graduate School of Art and Sciences, The University of Tokyo, 3-8-1, Komaba, Meguro-ku, Tokyo 153-8902, Japan. ${ }^{6}$ Department of Rehabilitation, Graduate School of Medicine, The University of Tokyo, 7-3-1, Hongo, Bunkyo-ku, Tokyo 113-8655, Japan

\section{Conflict of interest}

The authors declare that they have no conflict of interest.

\section{Publisher's note}

Springer Nature remains neutral with regard to jurisdictional claims in published maps and institutional affiliations.

Supplementary Information accompanies this paper at (https://doi.org/ 10.1038/s41398-018-0261-0).

Received: 1 March 2018 Revised: 26 July 2018 Accepted: 7 September 2018 Published online: 08 October 2018

\section{References}

1. Coyle, J. T., Tsai, G. \& Goff, D. Converging evidence of NMDA receptor hypofunction in the pathophysiology of schizophrenia. Ann. N. Y. Acad. Sci. 1003, 318-327 (2003).

2. Coyle, J. T. NMDA receptor and schizophrenia: a brief history. Schizophr. Bull. 38, 920-926 (2012)

3. Moghaddam, B. \& Krystal, J. H. Capturing the angel in "angel dust": twenty years of translational neuroscience studies of NMDA receptor antagonists in animals and humans. Schizophr. Bull. 38, 942-949 (2012).

4. Krystal, J. H. et al. Subanesthetic effects of the noncompetitive NMDA antagonist, ketamine, in humans. Psychotomimetic, perceptual, cognitive, and neuroendocrine responses. Arch. Gen. Psychiatry 51, 199-214 (1994).

5. Hashimoto, K. et al. Elevated glutamine/glutamate ratio in cerebrospinal fluid of first episode and drug naive schizophrenic patients. BMC Psychiatry 5, 6 (2005).

6. Brouwer, A., Luykx, J. J., van Boxmeer, L., Bakker, S. C. \& Kahn, R. S. NMDAreceptor coagonists in serum, plasma, and cerebrospinal fluid of schizophrenia patients: a meta-analysis of case-control studies. Neurosci. Biobehav. Rev. 37, 1587-1596 (2013).

7. Bustillo, J. R. et al. Increased glutamine in patients undergoing long-term treatment for schizophrenia: a proton magnetic resonance spectroscopy study at 3 T. JAMA Psychiatry 71, 265-272 (2014).

8. Bustillo, J. R. et al. 1H-MRS at 4 tesla in minimally treated early schizophrenia. Mol. Psychiatry 15, 629-636 (2010).

9. Akbarian, S. \& Huang, H. S. Molecular and cellular mechanisms of altered GAD1/GAD67 expression in schizophrenia and related disorders. Brain Res. Rev. 52, 293-304 (2006).

10. Eyles, D. W., McGrath, J. J. \& Reynolds, G. P. Neuronal calcium-binding proteins and schizophrenia. Schizophr. Res. 57, 27-34 (2002).

11. Gonzalez-Burgos, G. \& Lewis, D. A. GABA neurons and the mechanisms of network oscillations: implications for understanding cortical dysfunction in schizophrenia. Schizophr. Bull. 34, 944-961 (2008).

12. Rowland, L. M. et al. Medial frontal GABA is lower in older schizophrenia: a MEGA-PRESS with macromolecule suppression study. Mol. Psychiatry 21, 198-204 (2016).
13. Chiu, P. W. et al. In vivo gamma-aminobutyric acid and glutamate levels in people with first-episode schizophrenia: a proton magnetic resonance spectroscopy study. Schizophr. Res. 193, 295-303 (2018).

14. Whittington, M. A., Traub, R. D., Kopell, N., Ermentrout, B. \& Buhl, E. H. Inhibitionbased rhythms: experimental and mathematical observations on network dynamics. Int. J. Psychophysiol. 38, 315-336 (2000).

15. Gonzalez-Burgos, G., Cho, R. Y. \& Lewis, D. A. Alterations in cortical network oscillations and parvalbumin neurons in schizophrenia. Biol. Psychiatry 77, 1031-1040 (2015)

16. Cochran, S. M. et al. Induction of metabolic hypofunction and neurochemical deficits after chronic intermittent exposure to phencyclidine: differential modulation by antipsychotic drugs. Neuropsychopharmacology 28, 265-275 (2003).

17. Keilhoff, G., Becker, A., Grecksch, G., Wolf, G. \& Bernstein, H. G. Repeated application of ketamine to rats induces changes in the hippocampal expression of parvalbumin, neuronal nitric oxide synthase and CFOS similar to those found in human schizophrenia. Neuroscience 126, 591-598 (2004).

18. Rujescu, D. et al. A pharmacological model for psychosis based on N-methylD-aspartate receptor hypofunction: molecular, cellular, functional and behavioral abnormalities. Biol. Psychiatry 59, 721-729 (2006).

19. Behrens, M. M. et al. Ketamine-induced loss of phenotype of fast-spiking interneurons is mediated by NADPH-oxidase. Science 318, 1645-1647 (2007).

20. Morrow, B. A., Elsworth, J. D. \& Roth, R. H. Repeated phencyclidine in monkeys results in loss of parvalbumin-containing axo-axonic projections in the prefrontal cortex. Psychopharmacol. (Berl.) 192, 283-290 (2007).

21. Belforte, J. E. et al. Postnatal NMDA receptor ablation in corticolimbic interneurons confers schizophrenia-like phenotypes. Nat. Neurosci. 13, 76-83 (2010).

22. Gordon, J. A. Testing the glutamate hypothesis of schizophrenia. Nat. Neurosci. 13, 2-4 (2010).

23. Carlen, M. et al. A critical role for NMDA receptors in parvalbumin interneurons for gamma rhythm induction and behavior. Mol. Psychiatry 17, 537-548 (2012).

24. Fujihara, K. et al. Glutamate decarboxylase 67 deficiency in a subset of gabaergic neurons induces schizophrenia-related phenotypes. Neuropsychopharmacology 40, 2475-2486 (2015).

25. Brown, J. A. et al. Inhibition of parvalbumin-expressing interneurons results in complex behavioral changes. Mol. Psychiatry 20, 1499-1507 (2015).

26. Light, G. A. \& Näätänen, R. Mismatch negativity is a breakthrough biomarker for understanding and treating psychotic disorders. Proc. Natl Acad. Sci. USA 110, 15175-15176 (2013).

27. Light, G. A. \& Swerdlow, N. R. Future clinical uses of neurophysiological biomarkers to predict and monitor treatment response for schizophrenia. Ann. N. Y. Acad. Sci. 1344, 105-119 (2015).

28. Näätänen, R., Shiga, T., Asano, S. \& Yabe, H. Mismatch negativity (MMN) deficiency: a break-through biomarker in predicting psychosis onset. Int. J. Psychophysiol. 95, 338-344 (2015).

29. Näätänen, R., Todd, J. \& Schall, U. Mismatch negativity (MMN) as biomarker predicting psychosis in clinically at-risk individuals. Biol. Psychol. 116, 36-40 (2016).

30. Javitt, D. C., Steinschneider, M., Schroeder, C. E. \& Arezzo, J. C. Role of cortical Nmethyl-D-aspartate receptors in auditory sensory memory and mismatch negativity generation: implications for schizophrenia. Proc. Natl Acad. Sci. USA 93, 11962-11967 (1996).

31. Umbricht, D. et al. Ketamine-induced deficits in auditory and visual contextdependent processing in healthy volunteers: implications for models of cognitive deficits in schizophrenia. Arch. Gen. Psychiatry 57, 1139-1147 (2000).

32. Gil-da-Costa, R., Stoner, G. R., Fung, R. \& Albright, T. D. Nonhuman primate model of schizophrenia using a noninvasive EEG method. Proc. Natl Acad. Sci. USA 110, 15425-15430 (2013)

33. Shelley, A. M. et al. Mismatch negativity: an index of a preattentive processing deficit in schizophrenia. Biol. Psychiatry 30, 1059-1062 (1991).

34. Javitt, D. C. et al. Impairment of early cortical processing in schizophrenia: an event-related potential confirmation study. Biol. Psychiatry 33, 513-519 (1993).

35. Catts, S. V. et al. Brain potential evidence for an auditory sensory memory deficit in schizophrenia. Am. J. Psychiatry 152, 213-219 (1995).

36. Light, G. A. \& Braff, D. L. Mismatch negativity deficits are associated with poor functioning in schizophrenia patients. Arch. Gen. Psychiatry 62, 127-136 (2005).

37. Umbricht, D. \& Krljes, S. Mismatch negativity in schizophrenia: a meta-analysis. Schizophr. Res. 76, 1-23 (2005). 
38. Erickson, M. A., Ruffle, A. \& Gold, J. M. A meta-analysis of mismatch negativity in schizophrenia: from clinical risk to disease specificity and progression. Biol. Psychiatry 79, 980-987 (2016).

39. Umbricht, D. S., Bates, J. A., Lieberman, J. A., Kane, J. M. \& Javitt, D. C. Electrophysiological indices of automatic and controlled auditory information processing in first-episode, recent-onset and chronic schizophrenia. Biol. Psychiatry 59, 762-772 (2006).

40. Todd, J. et al. Deviant matters: duration, frequency, and intensity deviants reveal different patterns of mismatch negativity reduction in early and late schizophrenia. Biol. Psychiatry 63, 58-64 (2008)

41. Kaur, M. et al. MMN/P3a deficits in first episode psychosis: comparing schizophrenia-spectrum and affective-spectrum subgroups. Schizophr. Res. 130, 203-209 (2011).

42. Nagai, T. et al. Auditory mismatch negativity and P3a in response to duration and frequency changes in the early stages of psychosis. Schizophr. Res. 150 547-554 (2013).

43. Haigh, S. M., Coffman, B. A. \& Salisbury, D. F. Mismatch negativity in firstepisode schizophrenia: a meta-analysis. Clin. Eeg. Neurosci. 48, 3-10 (2017).

44. Shin, K. S. et al. Pre-attentive auditory processing in ultra-high-risk for schizophrenia with magnetoencephalography. Biol. Psychiatry 65, 1071-1078 (2009).

45. Atkinson, R. J., Michie, P. T. \& Schall, U. Duration mismatch negativity and P3a in first-episode psychosis and individuals at ultra-high risk of psychosis. Biol. Psychiatry 71, 98-104 (2012).

46. Jahshan, $\mathrm{C}$. et al. Automatic sensory information processing abnormalities across the illness course of schizophrenia. Psychol. Med. 42, 85-97 (2012).

47. Shaikh, M. et al. Reduced mismatch negativity predates the onset of psychosis. Schizophr. Res. 134, 42-48 (2012).

48. Perez, V. B. et al. Automatic auditory processing deficits in schizophrenia and clinical high-risk patients: forecasting psychosis risk with mismatch negativity. Biol. Psychiatry 75, 459-469 (2014).

49. Bodatsch, M. et al. Prediction of psychosis by mismatch negativity. Biol. Psychiatry 69, 959-966 (2011).

50. Nagai, T. et al. Reduced mismatch negativity is associated with increased plasma level of glutamate in first-episode psychosis. Sci. Rep. 7, 2258 (2017).

51. McGale, E. H., Pye, I. F., Stonier, C., Hutchinson, E. C. \& Aber, G. M. Studies of the inter-relationship between cerebrospinal fluid and plasma amino acid concentrations in normal individuals. J. Neurochem. 29, 291-297 (1977).

52. Alfredsson, G., Wiesel, F. A. \& Lindberg, M. Glutamate and glutamine in cerebrospinal fluid and serum from healthy volunteers--analytical aspects. J. Chromatogr. 424, 378-384 (1988)

53. Rowland, L. M. et al. Frontal glutamate and gamma-aminobutyric acid levels and their associations with mismatch negativity and digit sequencing task performance in schizophrenia. JAMA Psychiatry 73, 166-174 (2016).

54. Cardin, J. A. et al. Driving fast-spiking cells induces gamma rhythm and controls sensory responses. Nature 459, 663-667 (2009).

55. Sohal, V. S., Zhang, F., Yizhar, O. \& Deisseroth, K. Parvalbumin neurons and gamma rhythms enhance cortical circuit performance. Nature 459, 698-702 (2009).

56. Kwon, J. S. et al. Gamma frequency-range abnormalities to auditory stimulation in schizophrenia. Arch. Gen. Psychiatry 56, 1001-1005 (1999).

57. Brenner, C. A., Sporns, O., Lysaker, P. H. \& O'Donnell, B. F. EEG synchronization to modulated auditory tones in schizophrenia, schizoaffective disorder, and schizotypal personality disorder. Am. J. Psychiatry 160, 2238-2240 (2003).

58. Light, G. A. et al. Gamma band oscillations reveal neural network cortical coherence dysfunction in schizophrenia patients. Biol. Psychiatry $\mathbf{6 0}$ 1231-1240 (2006).

59. Teale, P. et al. Cortical source estimates of gamma band amplitude and phase are different in schizophrenia. Neuroimage 42, 1481-1489 (2008).
60. Vierling-Claassen, D., Siekmeier, P., Stufflebeam, S. \& Kopell, N. Modeling GABA alterations in schizophrenia: a link between impaired inhibition and altered gamma and beta range auditory entrainment. J. Neurophysiol. 99, 2656-2671 (2008).

61. Tsuchimoto, R. et al. Reduced high and low frequency gamma synchronization in patients with chronic schizophrenia. Schizophr. Res. 133, 99-105 (2011).

62. Kirihara, K., Rissling, A. J., Swerdlow, N. R., Braff, D. L. \& Light, G. A. Hierarchical organization of gamma and theta oscillatory dynamics in schizophrenia. Biol. Psychiatry 71, 873-880 (2012).

63. Spencer, K. M., Salisbury, D. F., Shenton, M. E. \& McCarley, R. W. Gamma-band auditory steady-state responses are impaired in first episode psychosis. Biol. Psychiatry 64, 369-375 (2008).

64. Wilson, T. W. et al. Cortical gamma generators suggest abnormal auditory circuitry in early-onset psychosis. Cereb. Cortex 18, 371-378 (2008).

65. Tada, M. et al. Differential alterations of auditory gamma oscillatory responses between pre-onset high-risk individuals and first-episode schizophrenia. Cereb. Cortex 26, 1027-1035 (2016).

66. Thuné, H., Recasens, M. \& Uhlhaas, P. J. The 40-Hz auditory steady-state response in patients with schizophrenia: a meta-analysis. JAMA Psychiatry 73, 1145-1153 (2016).

67. Koike, S. et al. A multimodal approach to investigate biomarkers for psychosis in a clinical setting: the integrative neuroimaging studies in schizophrenia targeting for early intervention and prevention (IN-STEP) project. Schizophr. Res. 143, 116-124 (2013)

68. Kobayashi, H., Nozaki, S. \& Mizuno, M. Reliability of the structured interview for prodromal syndromes Japanese version (SIPS-J). Jpn Bull. Soc. Psychiat 15 168-174 (2007). (In Japanese).

69. Matsuoka, K. \& Kim, Y. Japanese Adult Reading Test (JART). (Shinkou-lgaku Publishers, Tokyo, 2006). (In Japanese).

70. Matsuoka, K., Uno, M., Kasai, K., Koyama, K. \& Kim, Y. Estimation of premorbid IQ in individuals with Alzheimer's disease using Japanese ideographic script (Kanji) compound words: Japanese version of National Adult Reading Test. Psychiatry Clin. Neurosci. 60, 332-339 (2006).

71. Kay, S. R., Fiszbein, A. \& Opler, L. A. The positive and negative syndrome scale (PANSS) for schizophrenia. Schizophr. Bull. 13, 261-276 (1987).

72. Hall, R. C. Global assessment of functioning. A modified scale. Psychosomatics 36, 267-275 (1995).

73. Eguchi, S., Koike, S., Suga, M., Takizawa, R. \& Kasai, K. Psychological symptom and social functioning subscales of the modified Global Assessment of Functioning scale: reliability and validity of the Japanese version. Psychiatry Clin. Neurosci. 69, 126-127 (2015).

74. Inada, T. \& Inagaki, A. Psychotropic dose equivalence in Japan. Psychiatry Clin. Neurosci. 69, 440-447 (2015).

75. Delorme, A. \& Makeig, S. EEGLAB: an open source toolbox for analysis of single-trial EEG dynamics including independent component analysis. J. Neurosci. Methods 134, 9-21 (2004).

76. Michie, P. T., Innes-Brown, H., Todd, J. \& Jablensky, A. V. Duration mismatch negativity in biological relatives of patients with schizophrenia spectrum disorders. Biol. Psychiatry 52, 749-758 (2002).

77. Kappel, S. L., Looney, D., Mandic, D. P. \& Kidmose, P. Physiological artifacts in scalp EEG and ear-EEG. Biomed. Eng. Online 16, 103 (2017).

78. Nakazawa, K., Jeevakumar, V. \& Nakao, K. Spatial and temporal boundaries of NMDA receptor hypofunction leading to schizophrenia. NPJ Schizophr. 3, 7 (2017).

79. Nakao, K. \& Nakazawa, K. Brain state-dependent abnormal LFP activity in the auditory cortex of a schizophrenia mouse model. Front. Neurosci. 8, 168 (2014). 